\title{
AN ANALYSIS OF SURFACE AND GROWTH DifFERENCES IN Plants OF DifFERENT STAGES Using IMAge Processing
}

\author{
Faizur Rashid and Tamirat Delesa \\ Department of Computer Science, College of Computing and Informatics \\ Haramaya University, Ethiopia
}

\begin{abstract}
Genomes are main reason for growth and surface differences in the plants. All plants grow on basis of their different surrounding like soil, water, breed of seed, and climatic session. This paper attempts to find stem growth from birth to maturity level of selected plant using image processing technique. Few reasons have been observed commonly by the researchers that some plants could not grow sufficiently as to the other plants of similar breed and age. Images were taken of different stage of bean plant and images of some other plant samples were also included for better assessment. Researchers are trying to understand through their calculative analysis by image processing emulator in MATLAB to view its reasons. Suitable comparison technique is applied to detect their period of growth. Image processing methods like Restoration, stem or leaves spots, filtering, and plant comparison have applied in MATLAB. Those effects that are not supporting to grow the plants of their similar age group are matter to calculate scientifically later in the future. The observation would help for further support in medicinal science or agricultural science of plant and Bio-chemical.
\end{abstract}

\section{KEYWORDS}

Image Processing, Surface of Plants (SOP), Differences of Stage (DoS), Segmentation.

\section{INTRODUCTION}

Image processing is a method to do some processing of observation in digital images [10]. The processing can be applied by using different techniques of emulators in images and required output can be gained with its characteristics. The image can be calculated by applying its function $\mathrm{f}(\mathrm{x}, \mathrm{y})$, where $\mathrm{x}$ and $\mathrm{y}$ are two coordinates. These coordinates are called the intensity or grayscale of the image at that point [10] [16].

These coordinates indicate that each point is having some value of image and it can be identified through its location for any means [1][4].

The existing method plant disease and growth is made by open eye observation during the time. It is a time process with a large number of team members and finding the solution. It is tried here to detect the growth on its different stages using automatic system of image processing using plant leaves and roots. The automatic detection analysis method will help the agricultural science to find ironic solution.

Either in the case continuous monitoring is required that has been made in beans plant on the area of $3 \times 3$ meters soil structure to observe their root growth and development in the land of Harar 
Region in Ethiopia. Its growth identification is more easy using automatic system in image processing.

\subsection{Edge of Image}

Edge detection is a mathematical rule and described fundamentals that help to identify the stages of plants of their age [4][12]. Complete surface can be observed and determined by the processes and comparison is easy to determine from the different stages of plants in image processing [6]. Edge detection and segmentation is very important area in the field of image processing that helps to precede different levels on any images.

An edge is defined as the boundary between regions in an image, which helps with segmentation and object recognition. Any shadow or change on the originality of the image can be easily identified by the segmentation process with distinct change in the intensity of an image [2][3]. The growth of any plant is dependable on various resources like quality of soil, period of rain or watering on the plant, intensity of sun, weather quality whether moisture or dry, and any deceased or non-deceased.

The quality of edge detection is highly dependent on the light, intensity on object, edge density, and noise on the plant. Many corrections are possible by adjustment of some certain value on the images with edge detection and threshold value mechanism [3] [8]. Some of the applications like Photoshop and Corel Draw help the users to adjust the image by using threshold techniques but it is hard to identify the originality of the image in auto-adjustment mechanism. The evaluation of categorical data was made using different techniques like Canny edge detection, skin segmentation [10], and morphological operation to detect the growth differences of Bean plant.

\section{Methodology}

The methodology being proposed by the author was staged in given below diagram that has been partly inherited [1], and shown here in Figure 2.1.

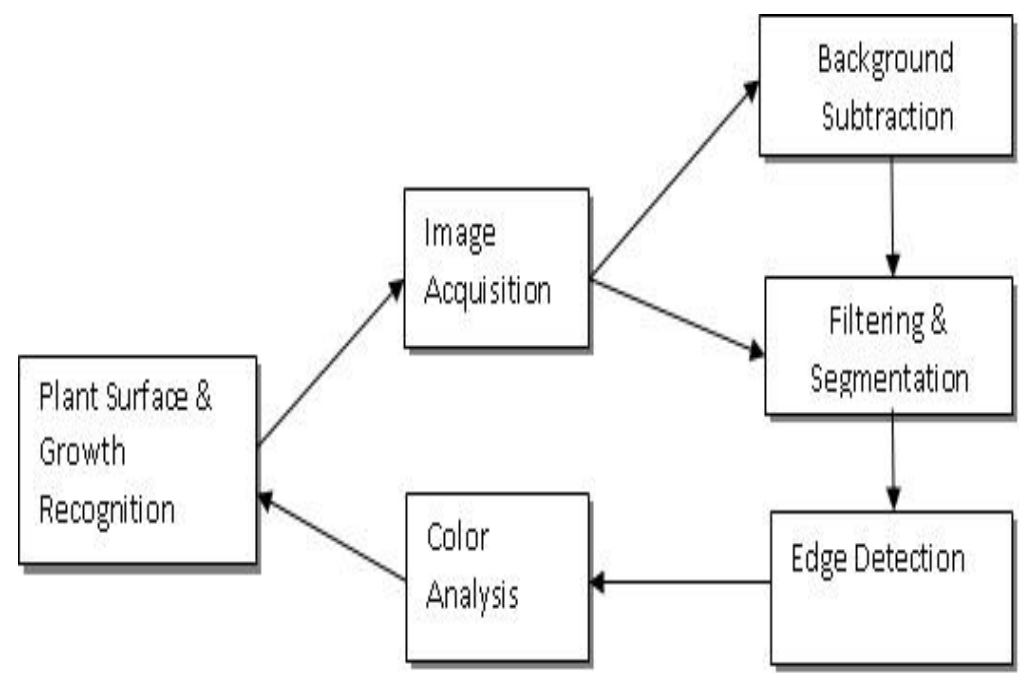

Figure 2.1 Steps of Plant Identification

The steps involved in identification of surface of plant growth in their quality and differences of Stages (DoS) in the growth. The frame in Figure 2.1 shows the plant acquisition, filtering and 
segmentation process, color of plant leaf surface analysis, and Canny edge detection. Finally, plant quality and growth were identified.

\subsection{Image Acquisition}

This was done with the help of Canon digital camera that loaded and saved through built in software. The images were generally saved in .jpg format that can be transmitted into different format like .tif, .img, .bmp, or binary manner [5].

Basically, the images were captured by the camera in RGB format by default, which was taken by the author for its further assessment.

The images that have been obtained in normal form of RGB may not be directly used for identification and classification due to some certain factors like noise, resolution quality artifacts, poor light, or very contrast light.

\subsection{Sample Plant Images}

Almost 45 images have been tested of different plants which are not possible to display in this small size of scripted form. The authors tried to display some of the images in integrated object. There are way of integration in MATLAB by using imgadd() command to combine the images into one form for object visibility in compared manner.

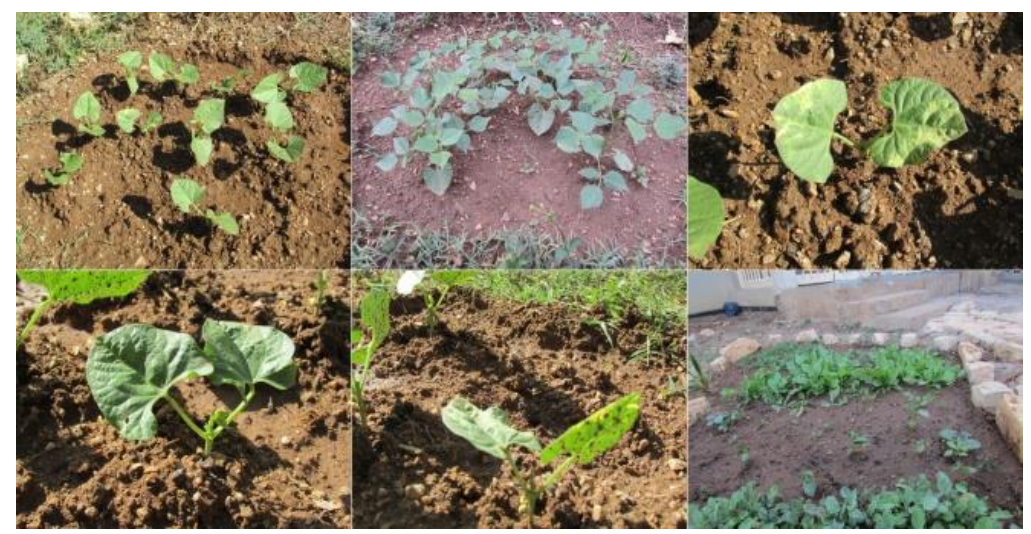

Figure 2.2 Combined RGB Images

The RGB image means a true color image that carry Red, Grey, and Blue color combination into matrix form. Similar size of the matrix is required to asses and each represents the image color in terms of pixels.

\subsubsection{Field data}

Images were collected in a period of 2 month from September'2018 to October'2018. Plants were selected randomly to snap and sampled for process. Morphological status was recorded on almost 7 times for different stage of the plants. The growth estimation were observed by taking a standard variable for different stage (S) and for plant (P), categorized in 3 levels S1, S2, S3 and $\mathrm{P} 1, \mathrm{P} 2$, and $\mathrm{P} 3$ within 3 months period of time. 
The height of stem were measured in S1 ( $4^{\text {rd }}$ week), S2 ( $7^{\text {th }}$ week $)$, and S3 (12 ${ }^{\text {th }}$ week) level for different plant P1, P2, and P3.The measurement were taken directly through geometrical scale from the level of soil to the top of the plant. The table below shows the growth level of the plants in table 1 .

Table 1. Growth level estimation of the plants in different stage

\begin{tabular}{|l|c|c|c|}
\hline \multirow{2}{*}{ Plant Samples } & \multicolumn{3}{|c|}{ Growth level in different Stages in inch(s) } \\
\cline { 2 - 4 } & $\mathrm{S} 1\left(4^{\text {rd }}\right.$ week $)$ & $\mathrm{S} 2\left(7^{\text {th }}\right.$ week $)$ & $\mathrm{S} 3\left(12^{\text {th }}\right.$ week $)$ \\
\hline P1 & 2.4 & 5.3 & 15.6 \\
\hline P2 & 2.0 & 4.9 & 13.2 \\
\hline P3 & 1.8 & 4.2 & 12.7 \\
\hline
\end{tabular}

\subsection{Grey Scale Plant Images}

Grey colour images are monochromic color images which contains only one combined color of black and white. The conversion from RGB to grey scale is required gamma extension [9][10]. Figure 2.3 shows the younger and older images of Plants.
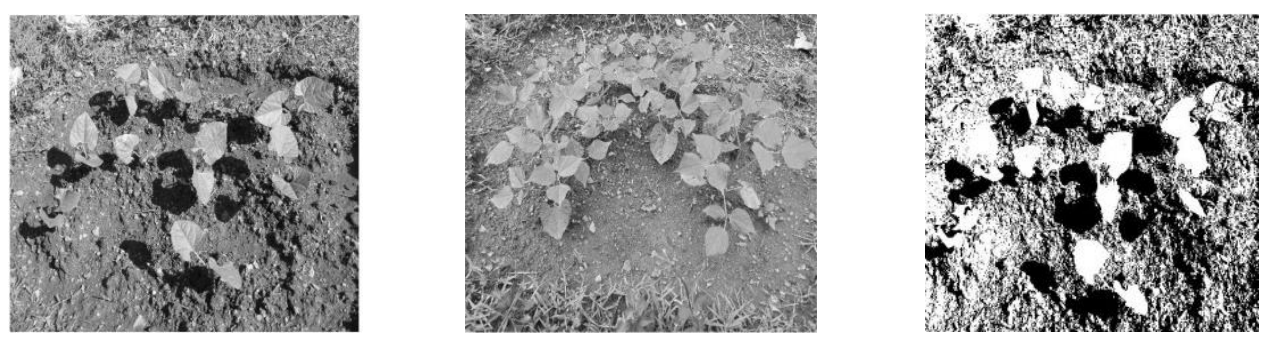

Figure 2.3 Grey Images of Plant of 16 bit

\subsection{Binary Image of Plant}

As the images are considered using 1 bit, binary image becomes applicable, which is based on 1s and 0s. Binary images are used to segmentation and pattern recognition where main task is to binaries' the image [10]. This Figure 2.4 is the way of changing luminance quantization of an image using $0=$ black, $1=$ dark grey, $2=$ light grey and $3=$ white with its value.

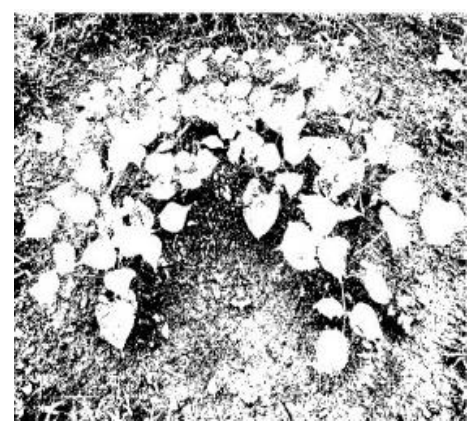

Figure 2.4 Binary Image 


\subsection{Filtering}

The purpose of smoothing is to reduce noise using geometric features and improve the visual quality of the image. Smoothing operation can be expressed as filtering. Here median filer is being used. This filters the high intensity sharp components by computing the median of the neighbourhood [8][13].

\subsubsection{Median Filter}

The set of number $m$, is finding the medium of two sets of numbers less and greater than $m$ consecutively 7][11][15]. It uses the neighbourhood value to calculate in a series of pixel and replaces the value of the pixel in matrix of the value of image [10][11][15]. The expression of Median Filter Is

$$
\mathrm{S}_{\mathrm{ij}}=1 / \mathrm{M} \sum_{(n, m) \in S} f i j
$$

Where $S$ is containing $n \times m$ pixel whose centre is located in the range of $(i, j)$ and $M$ is the total number of pixels in the image that calculate the median of the image.

The median filter is important to remove isolated random noise without blurring. Median filter takes out little better result than other filtering process [10]. Below in figure 2.5 shows the median filter result.

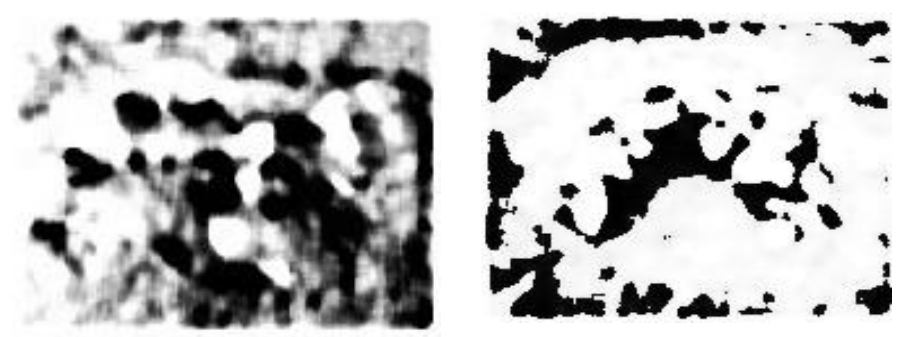

Figure 2.5 Filtered Images

\section{SEGMENTATION}

Segmentation allows set of raw components to be generated that is region of pixels with similar intensity of values or set of lines obtained by isolating the edges of an image and computed by locating regions where there is significance difference in the intensity. Different regions of image are divided with this process [14][15]. It is used to analyse the separate feature of pre-determined type of background. This is broadly known for two properties in an image, (i) Similarity (ii) discontinuity.

Firstly, the image is having gray level of predetermined range in different regions in image. Secondly, the sudden change of the value of gray level image is observed in another segmented region, which is also known as edge detection. Edge detection is obvious to observe the edges of image to analyse the growth of the plant. This is being applied by canny edge detector mechanism in this work [10]. 


\section{Canny Edge Detector Method}

This is also known as Optimal Edge Detector Algorithm. Canny vision was to enhance the images into many different forms with following properties [10]

a) Reduce the amount of noise speckles within the image and remove the very high frequency components.

b) Creating two images, one containing the gradient magnitude $\mathrm{G}$, and another containing the orientation $\theta(G)$. Means, keep lower variation between original and processed image.

c) Certain minimum threshold value above gradient magnitude is detected to find major edges.

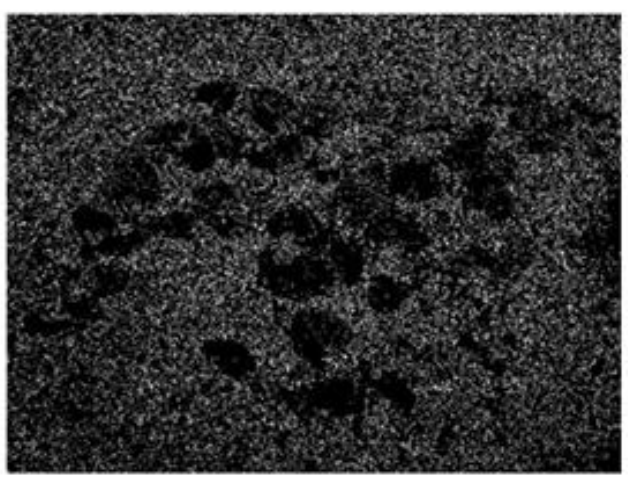

(a) Canny edge older plant

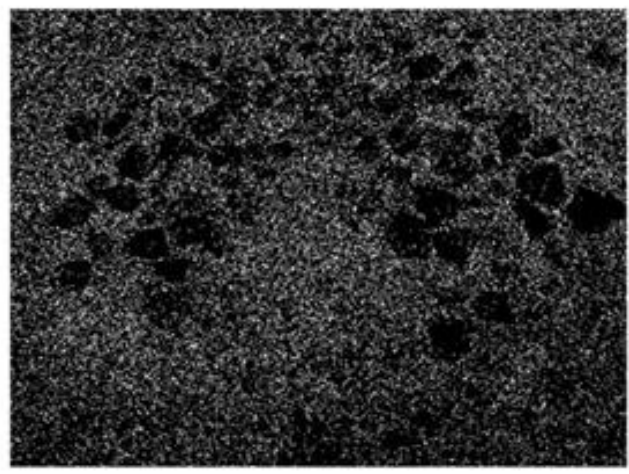

(b) Canny edge Younger plant

Canny edge detector were applied using MATLAB in older and younger plant. Code of MATLAB is as follows,

e=edge(g,'canny',[],1);

subplot $(2,2,4)$;

figure, imshow(e);

Here, $e$ will store the two dimensional grey image that stored in $g$. It will acquire an area that is need to display the edged image and lastly displaying the image in cascaded manner.

\section{CONCLUSION}

Basically leaves of plants were analysed and entire expedients done in MATLAB version 14a. Additionally, some of the images were cross tested in Corel Photo Paint to observe the display quality of image output. In cross observation, concluded that MATLAB is better option to the image output in conversion, effects or transmission. Firstly, it was observed that binary value differences were found in the binary converted image in the matrix positions. Secondly, the points of higher number of the detected edges approves, the higher the age and grown plant. Background color detection was tried to observe the affected plant leaf, and observed that changed color from the originated leaves are infected by some diseases and causes the competitive growth among the others. This disease could be identified in other step of work with the help of specialist.

All the script of MATAB has not been written in this paper, only some of the samples have been mentioned. 


\section{REFERENCES}

[1]. Y. Rouphael, A.H. Mouneimne, A. Ismael, E. Mendoza, C.M. Rivera, "Modeling Individual leaf based on leaf length and width measurement", 48, pages 9-15, 2010.

[2]. C. Feng, Quwang, Yunming Du, "Face Detection Based on Membership and Geometry Feature", Harbin, 31(8), pages. 84-87, 2007.

[3]. M. Vidal Naquet and S. Ullman, "Object Recognition with informative Features and Linear Classification", IEEE, International Conference on Computer Vision (ICCV), Pages 281-288, October 2003.

[4]. Mohammad A, Yassin H., Fekadu D., Hussen S., "Effect of flower bud removal growth and yield of anchote root accession", 1, pages 7-13, 2013.

[5]. J Sivic and A. Zisserman, "Efficient Visual Search of Videos cast as text Retrieval" Volume 31, pages 591-606, April 2009.

[6]. A.S. Samra, S.EI, Taweel Gad Allah, and R.M. Ibrahim, "Face Recognition using Wavelet Transform, Fast Fourier Transformation and discrete Cosine Transform in Circuit and System", IEEE, 46th Midwest Symposium, Volume 1, Pages 272-275, Dec.2003.

[7]. Huchuan Lu and Hui Lin, "Gender Recognition using Ad boosted Feature", 3rd International Conference on Natural Computation, Volume 2, Pages 646-650, Aug. 2007.

[8]. Zisheng Li, J. Imai, and M. Kaneko, "Robust Face Recognition using Block Based bag of Words", 20th International Conference on Pattern Recognition (ICPR), Pages 1285-1288, Aug 2010.

[9]. Jonathan M. Blackledge, "Digital Image Processing", Harwood Publishing, Chinchester, West Sussex, 2005.

[10]. Rouphael Y., Rivera C.M., Buttaro D., Colla G., Gonella M., "Simple and accurate allometric model for leaf area estimation".53, pages 1-8, 2015.

[11]. J.G. Wang E. Sung, J. Li and W.Y. Yau, Boosting dense sift descriptors and shape contexts of face images for gender recognition. IEEE Conference on Computer Vision and Pattern Recognition Workshop, Pages 96-102, June 2010.

[12]. L.L. Yu D. Gonzalez-Jimenez, J.L. Alba- Castro and P. Dago-Casas, "Single and cross- database benchmarks for gender classification under unconstrained setting", IEEE International Conference on Computer Vision Workshop, Pages 2152-2159. Nov'2011.

[13]. Oana G. Cula and Krstin, J. Dana, 3D Texture recognition using bidirectional feature histograms, Volume-59, 2004.

[14]. H. Singh and S.K. Pandey, "A simple cost effective method for leaf area estimation", pages 1-6, 2011.

[15]. Steven L Edins, “Image Processing” 1993. 